NDA-FP-48

May 1998

\title{
CAN QUANTUM-CORRECTED BTZ BLACK HOLE ANTI-EVAPORATE?
}

\author{
SHIN'ICHI NOJIRI" and SERGEI D. ODINTSOV \\ Department of Mathematics and Physics \\ National Defence Academy \\ Hashirimizu Yokosuka 239, JAPAN \\ ه Tomsk Pedagogical University \\ 634041 Tomsk, RUSSIA
}

\begin{abstract}
Kaluza-Klein reduction of 3D gravity with minimal scalars leads to 2D dilaton-Maxwell gravity with dilaton coupled scalars. Evaluating the oneloop effective action for dilaton coupled scalars in large $N$ and $s$-wave approximation we apply it to study quantum evolution of BTZ black hole. It is shown that quantum-corrected BTZ BH may evaporate or else anti-evaporate similarly to 4D Nariai BH as is observed by Bousso and Hawking. Instable higher modes in the spectrum indicate also the possibility of proliferation of BTZ BH.
\end{abstract}

PACS: 04.60.-m, 04.70.Dy, 11.25.-w

\footnotetext{
1 e-mail : nojiri@cc.nda.ac.jp

2 e-mail : odintsov@tspi.tomsk.su
} 
1. BTZ black hole (BH) [5 attracts a lot of attention due to various reasons. In particulary, it is related via T-duality with a class of asymptotically flat black strings [6] and via U-duality it is related [B] with 4D and 5D stringy BHs [9] which are asymptotically flat ones. Hence, microscopically computing the entropy of BTZ BH 10 may be applied via duality relations for the computing of entropy for higher dimensional BHs. This fact is quite remarkable as above types of BHs look completely different from topological, dimensional or space-time points of view.

BTZ BH being locally $\mathrm{AdS}^{3}$ without curvature singularity may be considered as a prototype for general CFT/AdS correspondence [11]. Indeed, 3D gravity has no local dynamics but $\mathrm{BH}$ horizon induces an effective boundary (actually, 2D WZWN model). Hence,quantum studies around BTZ BH may help in better understanding of above correspondence.

In the present letter we study quantum dynamics of BTZ BH due to 3D minimal scalars. We work in large $N$ and $s$-wave approach where minimal scalars are described as 2D dilaton coupled scalars. The quantum corrected version of BTZ $\mathrm{BH}$ is found. It is shown that it can evaporate or antievaporate in accordance with recent observation by Bousso and Hawking [3] made for $4 \mathrm{D}$ SdS BH. Higher modes perturbations in the quantum spectrum are also briefly discussed.

2. Three dimensional Einstein gravity with the cosmological term has the exact rotating black hole solution [5] and the solution can be regarded as an exact solution of string theory [6]. In this section, we consider the circle reduction of three dimensional Einstein gravity to two dimensional one. Now we identify the coordinates as $x^{1}=t, x^{2}=r, x^{3}=\phi$. Here $t, r$ and $\phi$ are the coordinates of time, radius, and angle. We now assume that all the fields do not depend on $x^{3}$. Then, under the following infinitesimal coordinate transformation $x^{3} \rightarrow x^{3}+\epsilon\left(x^{1}, x^{2}\right)$, the metric tensors transform as follows;

$$
\delta g^{(3) 3 \alpha}=\delta g^{(3) \alpha 3}=g^{(3) \alpha \beta} \partial_{\beta} \epsilon, \quad \delta g^{(3) 33}=2 g^{(3) 3 \alpha} \partial_{\alpha} \epsilon, \quad \delta g^{(3) \alpha \beta}=0
$$

Here $\alpha, \beta=1,2$. Eq.(1) tells that we can identify the gauge vector field $A_{\alpha}$ with $g^{(3) 3 \alpha}=g^{(3) \alpha \beta} A_{\beta}$. Eq.(1) also tells that the metric tensor $g^{(3) \mu \nu}$ $(\mu, \nu=1,2,3)$ can be parametrized a la Kaluza-Klein as

$$
g^{(3) \mu \nu}=\left(\begin{array}{cc}
g^{\alpha \beta} & g^{\alpha \gamma} A_{\gamma} \\
g^{\beta \gamma} A_{\gamma} & \mathrm{e}^{2 \phi}+g^{\gamma \delta} A_{\gamma} A_{\delta}
\end{array}\right) .
$$


Then we find

$$
\begin{aligned}
g^{(3)} & \equiv \frac{1}{\operatorname{det} g^{(3) \mu \nu}}=g \mathrm{e}^{-2 \phi} \quad\left(g \equiv \frac{1}{\operatorname{det} g^{\mu \nu}}\right) \\
g_{\mu \nu}^{(3)} & =\left(\begin{array}{cc}
g_{\alpha \beta}+\mathrm{e}^{-2 \phi} A_{\mu} A_{\nu} & -A_{\gamma} \\
-A_{\gamma} & \mathrm{e}^{-2 \phi}
\end{array}\right) .
\end{aligned}
$$

In the following the quantities in three dimensions are denoted by the suffix "(3)" and the quantities without the suffix are those in two dimensions unless we mention. In the parametrization (2), curvature has the following form

$$
R^{(3)}=R+\square \phi-\partial_{\alpha} \phi \partial^{\alpha} \phi-\frac{1}{4} \mathrm{e}^{-2 \phi} F_{\alpha \beta} F^{\alpha \beta} .
$$

Here $F_{\alpha \beta}$ is the field strength: $F_{\alpha \beta}=\partial_{\alpha} A_{\beta}-\partial_{\beta} A_{\alpha}$. Then the action $S$ of the gravity with cosmological term and with $N$ free scalars $f_{i}(i=1, \cdots, N)$ in three dimensions is reduced as

$$
\begin{aligned}
S & =\frac{1}{4 \pi^{2}} \int d^{3} x \sqrt{g^{(3)}}\left\{\frac{1}{8 G}\left(R^{(3)}+\Lambda\right)+\frac{1}{2} \sum_{i=1}^{N} \partial_{\mu} f_{i} \partial^{\mu} f^{i}\right\} \\
& \sim \frac{1}{2 \pi} \int d^{2} x \sqrt{g} \mathrm{e}^{-\phi}\left\{\frac{1}{8 G}\left(R-\frac{1}{4} \mathrm{e}^{-2 \phi} F_{\alpha \beta} F^{\alpha \beta}+\Lambda\right)+\frac{1}{2} \sum_{i=1}^{N} \partial_{\alpha} f_{i} \partial^{\alpha} f^{i}\right\}(6)
\end{aligned}
$$

Note that the contribution from the second and third terms in (50) becomes the total derivative which maybe neglected. One BH solution for the classical action is given by [5]

$$
\begin{aligned}
& d s^{2}=-\mathrm{e}^{2 \rho_{c l}} d t^{2}+\mathrm{e}^{-2 \rho_{c l} l} d x^{2}, \quad \mathrm{e}^{2 \rho_{c l}}=-M+\frac{x^{2}}{l^{2}}+\frac{J^{2}}{4 x^{2}} \\
& \mathrm{e}^{-\phi}=\mathrm{e}^{-\phi_{c l}}=\mathrm{e}^{-\phi_{0}} x
\end{aligned}
$$

Here $\frac{1}{l^{2}}=\frac{\Lambda}{2} . M$ is the parameter related to the black hole mass and $J$ is that of the angular momentum in 3D model or the electromagnetic charge in the 2D model. The extremal limit is given by $J^{2}=l^{2} M^{2}$. In order to consider this limit, we change the coordinates as follows

$$
x^{2}=\frac{l^{2} M(1+\epsilon \tanh r)}{2}, \quad t=\frac{l}{\epsilon \sqrt{2}} \tau .
$$


Then $r \rightarrow+\infty$ corresponds to outer horizon and $r \rightarrow-\infty$ corresponds to inner horizon. Taking the limit of $\epsilon \rightarrow 0$, we obtain

$$
d s^{2}=\frac{l^{2} M}{4 \cosh ^{2} r}\left(d \tau^{2}-d r^{2}\right), \quad \mathrm{e}^{-\phi}=l \mathrm{e}^{-\phi_{0}} \sqrt{\frac{M}{2}} .
$$

Note that $\phi$ becomes a constant in this limit.

3. We will discuss now the quantum corrections induced by $N$ free conformally invariant dilaton coupled scalars $f_{i}::^{3}$

$$
S^{f}=-\frac{1}{2} \int d^{2} x \sqrt{-g} \mathrm{e}^{-\phi} \sum_{i=1}^{N}\left(\nabla f_{i}\right)^{2} .
$$

This action represents reduction of 3D minimal scalars action. We present now the two-dimensional metric as $d s^{2}=\mathrm{e}^{2 \sigma} \tilde{g}_{\mu \nu} d x^{\mu} d x^{\nu}$, where $\tilde{g}_{\mu \nu}$ is the flat metric. Then the total quantum action coming from quantum scalars is given as (we work in large $N$ approximation what justifies the neglecting of proper quantum gravitational corrections):

$$
\Gamma=W+\Gamma\left[1, \tilde{g}_{\mu \nu}\right]
$$

where $W$ is conformal anomaly induced effective action [1, 2] (actually, scaledependent part) and $\Gamma\left[1, \tilde{g}_{\mu \nu}\right]$ is one-loop conformally invariant effective action for scalars (10) calculated on flat $2 \mathrm{D}$ metric.

Following [2] ( using conformal anomaly of dilaton coupled scalar [1, 2, 12]) we obtain

$$
W=-\frac{1}{2} \int d^{2} x \sqrt{-g}\left[\frac{N}{48 \pi} R \frac{1}{\Delta} R-\frac{N}{16 \pi} \nabla^{\lambda} \phi \nabla_{\lambda} \phi \frac{1}{\Delta} R+\frac{N}{8 \pi} \phi R\right] .
$$

We are left with the calculation of the conformally invariant part of the effective action $\Gamma\left[1, \tilde{g}_{\mu \nu}\right]$. This term has to be calculated on the flat space, after that the general covariance should be restored. It is impossible to do such calculation in closed form. We apply Schwinger-De Witt technique in order to get this effective action as local curvature expansion.

\footnotetext{
${ }^{3}$ Quantum corrections due to matter near BTZ black hole have been also studied in [7].
} 
Using the results of ref. [2], one can obtain:

$$
\Gamma\left[1, \tilde{g}_{\mu \nu}\right]=\frac{N}{16 \pi} \int d^{2} x \sqrt{-\tilde{g}}\left(-\tilde{\nabla}_{\mu} \phi \tilde{\nabla}^{\mu} \phi\right) \ln \mu^{2}+\cdots
$$

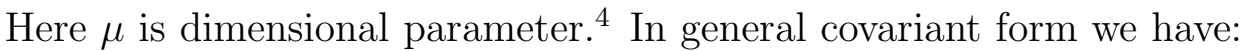

$$
\Gamma\left[1, g_{\mu \nu}\right]=-\frac{N}{16 \pi} \int d^{2} x \sqrt{-g} \nabla_{\mu} \phi \nabla^{\mu} \phi \ln \mu^{2}+\cdots
$$

This action is conformally invariant as it should be in accordance with Eq.(11).

4. In the study of quantum corrected $\mathrm{BH}$ we work in the conformal gauge: $g_{ \pm \mp}=-\frac{1}{2} \mathrm{e}^{2 \rho}, \quad g_{ \pm \pm}=0$. We are going to study (in)stability of black holes in the same way as in four dimensions [4]. by

The equations of motion with account of quantum corrections are given

$$
\begin{aligned}
0= & \frac{1}{8 G} \mathrm{e}^{-\phi}\left(-\partial_{ \pm} \rho \partial_{ \pm} \phi-\frac{1}{2} \partial_{ \pm}^{2} \phi+\frac{1}{2}\left(\partial_{ \pm} \phi\right)^{2}\right)+\frac{N}{12}\left(\partial_{ \pm}^{2} \rho-\partial_{ \pm} \rho \partial_{ \pm} \rho\right) \\
& +\frac{N}{8}\left\{\left(\partial_{ \pm} \phi \partial_{ \pm} \phi\right) \rho+\frac{1}{2} \frac{\partial_{ \pm}}{\partial_{\mp}}\left(\partial_{ \pm} \phi \partial_{\mp} \phi\right)\right\} \\
& +\frac{N}{8}\left\{2 \partial_{ \pm} \rho \partial_{ \pm} \phi-\partial_{ \pm}^{2} \phi\right\}+t^{ \pm}\left(x^{ \pm}\right)+\frac{N}{8}\left(\partial_{ \pm} \phi \partial_{ \pm} \phi\right) \ln \mu^{2} \\
0= & \frac{1}{8 G} \mathrm{e}^{-\phi}\left(4 \partial_{+} \partial_{-} \phi-4 \partial_{+} \phi \partial_{-} \phi-\Lambda \mathrm{e}^{2 \rho}+2 \mathrm{e}^{-2 \phi-2 \rho}\left(F_{+-}\right)^{2}\right) \\
& -\frac{N}{12} \partial_{+} \partial_{-} \rho-\frac{N}{16} \partial_{+} \phi \partial_{-} \phi+\frac{N}{8} \partial_{+} \partial_{-} \phi \\
0 & \frac{1}{8 G} \mathrm{e}^{-\phi}\left(4 \partial_{+} \partial_{-} \rho+\frac{\Lambda}{2} \mathrm{e}^{2 \rho}+3 \mathrm{e}^{-2 \phi-2 \rho}\left(F_{+-}\right)^{2}\right) \\
& -\frac{N}{16} \partial_{+}\left(\rho \partial_{-} \phi\right)-\frac{N}{16} \partial_{-}\left(\rho \partial_{+} \phi\right)-\frac{N}{8} \partial_{+} \partial_{-} \rho-\frac{N}{4} \partial_{+} \partial_{-} \phi \ln \mu^{2} \\
0 & \partial_{ \pm}\left(\mathrm{e}^{-3 \phi-2 \rho} F_{+-}\right) .
\end{aligned}
$$

Eq.(18) can be integrated to give

$$
\mathrm{e}^{-3 \phi-2 \rho} F_{+-}=B \text { (constant) . }
$$

\footnotetext{
4 Note that more exactly, one should write $\ln \frac{L^{2}}{\mu^{2}}$ where $L^{2}$ is covariant cut-off parameter.
} 
We now assume $\phi$ is constant as in the classical extremal solution (9) even when we include the quantum correction: $\phi=\phi_{0}$ (constant). Then we obtain

$$
B^{2}=\frac{\Lambda \mathrm{e}^{-4 \phi_{0}}}{2}, \quad R=R_{0} \equiv-\frac{4 \Lambda \mathrm{e}^{-\phi_{0}}}{\mathrm{e}^{-\phi_{0}}-\frac{G N}{4}} .
$$

Quantum corrected extremal (static) solution corresponding to the classical one (9) can be written as follows:

$$
\mathrm{e}^{2 \rho}=\mathrm{e}^{2 \rho_{0}} \equiv \frac{2 C}{R_{0}} \cdot \frac{1}{\cosh ^{2}(r \sqrt{C})} .
$$

Here $r=\frac{x^{+}-x^{-}}{2}$ and $C$ is an integration constant.

In order to investigate the (in)stability in the above solution, we consider the perturbation around the extremal solution:

$$
\rho=\rho_{0}+\epsilon R, \quad \phi=\phi_{0}+\epsilon S .
$$

Following Bousso and Hawking's argument[3], we neglect the second term in (12). Then (16) and (17) (and (19)) lead to the following equations:

$$
\begin{aligned}
0= & \frac{1}{8 G} \mathrm{e}^{-\phi_{0}}\left\{4 \partial_{+} \partial_{-} S-\Lambda \mathrm{e}^{2 \rho_{0}}(2 R-S)+2 B^{2} \mathrm{e}^{2 \rho_{0}+4 \phi_{0}}(2 R+3 S)\right\} \\
& -\frac{N}{12} \partial_{+} \partial_{-} R+\frac{N}{8} \partial_{+} \partial_{-} S \\
0= & \frac{1}{8 G} \mathrm{e}^{-\phi_{0}}\left\{4 \partial_{+} \partial_{-} R-4 \partial_{+} \partial_{-} \rho_{0} S+\frac{\Lambda}{2} \mathrm{e}^{2 \rho_{0}}(2 R-S)\right. \\
& \left.+3 B^{2} \mathrm{e}^{2 \rho_{0}+4 \phi_{0}}(2 R+3 S)\right\}-\frac{N}{8} \partial_{+} \partial_{-} R-\frac{N}{4} \ln \mu^{2} \partial_{+} \partial_{-} S .
\end{aligned}
$$

We now assume $R$ and $S$ have the following form

$R(t, r)=P \cosh (t \alpha \sqrt{C}) \cosh ^{\alpha} r \sqrt{C}, S(t, r)=Q \cosh (t \alpha \sqrt{C}) \cosh ^{\alpha} r \sqrt{C}$.

Then (23) and (24) become algebraic equations and the condition that there is non-trivial solution for $Q$ and $P$ is given by

$$
\begin{aligned}
F^{B T Z}(A) \equiv & \mathrm{e}^{-2 \phi_{0}}\left(\frac{2 R_{0}}{C} A+4 \Lambda\right)^{2}-\left(\frac{G N R_{0}}{C} A\right)^{2} \\
& +\frac{G N R_{0}}{3 C} A\left\{\mathrm{e}^{-\phi_{0}}\left(-\frac{R_{0}}{2}+4 \Lambda\right)-G N\left(\ln \mu^{2}\right) \frac{R_{0}}{C} A\right\}=0(.26)
\end{aligned}
$$


Here $A=\frac{\alpha(\alpha-1) C}{4} \cdot \mathrm{e}^{-\phi}$ is identified with the radius in $3 \mathrm{D}$ model, so horizon is given by the condition

$$
\nabla \sigma \cdot \nabla \sigma=0
$$

Substituting (25) into (27), we find the horizon is given by $r= \pm \alpha t$. Therefore on the horizon, we obtain

$$
S(t, r(t))=Q \cosh ^{1+\alpha} t \alpha \sqrt{C}
$$

This tells that the system is unstable if there is a solution $0>\alpha>-1$, i.e., $0<A<\frac{C}{2}$. On the other hand, the perturbation becomes stable if there is a solution where $\alpha<-1$, i.e., $A>\frac{C}{2}$. The radius of the horizon $r_{h}$ is given by

$$
r_{h}=\mathrm{e}^{\sigma}=\mathrm{e}^{-\left(\phi_{0}+\epsilon S(t, r(t))\right)} .
$$

Let the initial perturbation is negative $Q<0$. Then the radius shrinks monotonically, i.e., the black hole evaporates (due to quantum effects) in case of $0>\alpha>-1$. On the other hand, the radius increases in time and approaches to the extremal limit asymptotically: $S(t, r(t)) \rightarrow Q \mathrm{e}^{(1+\alpha) t|\alpha| \sqrt{C}}$ in case of $\alpha<-1$. The latter case corresponds to the quantum anti-evaporation of Nariai black hole observed by Bousso and Hawking [3] in case of 4D BH with minimal scalars (for the case of conformal matter induced anti-evaporation, see[4]). In the classical limit $\mathrm{e}^{-2 \phi_{0}} \gg N, A=\frac{C}{2}$, what tells that there does not occur any kind of the radiation in the solution.

Eq.(26) can be solved with respect to $A$,

$$
\begin{aligned}
\frac{A}{C}= & {\left[-4096-2176 g+48 g^{2} \pm\left\{18939904 g+4321280 g^{2}\right.\right.} \\
& -208832 g^{3}+2304 g^{4}+\left(786432 g-49152 g^{2}\right. \\
& \left.\left.\left.+768 g^{3}\right) \ln \mu^{2}\right\}^{\frac{1}{2}}\right] \times\left\{2\left(-4096+16 g^{2}+192 g^{2} \ln \mu^{2}\right)\right\}^{-1} \\
\sim & \frac{1}{2} \pm \frac{3 \sqrt{2}}{4} g^{\frac{1}{2}}+\mathcal{O}(g)
\end{aligned}
$$

Here $g \equiv 8 G N \mathrm{e}^{\phi_{0}}$. Note that there are two solutions near the classical limit $g \rightarrow 0$, which correspond to stable and instable modes, respectively. It might be surprizing that there is an instable mode since the extremal solution is usually believed to be stable. The global behavior of $\frac{A}{C}$ is given in Figures. 
In Fig.1, the vertical line corresponds to $\frac{A}{C}$ and the horizontal one to $g$ when $\ln \mu^{2}=1$. There is a singularity when $g \sim 0.4376$. The singularity occurs when the denominator in (30) vanishes. In the range from $g=0$ to near the singularity, there coexist the stable and instable modes. In Fig.2a and 2b, we present the global behavior of two modes as a function of $g \equiv 8 G N \mathrm{e}^{\phi_{0}}$ and $\ln \mu^{2}$. Fig.2a corresponds to the stable mode and $2 \mathrm{~b}$ to the instable one.

5. In the recent study by Bousso 13 it has been proposed the possibility that de Sitter space proliferates. Consider Schwarzschild de Sitter (SdS) BH in an extremal regime (Nariai BH). Topology of Nariai space is $S^{1} \times S^{2}$, therefore the solution can describe a handle. If there is a perturbation, multiple pair of the cosmological and black hole horizons are formed. After that $\mathrm{BHs}$ evaporate and BHs horizons tend to zero. Therefore the handle is separated into several pieces, which are copies of the de Sitter spaces. The above analysis has been supported by the study of higher modes perturbations in the spectrum of Nariai BH.

The metric (9) in the extremal limit or its quantum analogue (21) has the same spacetime structure as the Nariai solution except its signature. Therefore there is a possibility of the multiple production of universes from the extremal soltion in 3D BTZ BH also.

Let us define new coordinate $\theta$

$$
\sin \theta=\tanh (r \sqrt{C})
$$

Then metric corresponding to (21) becomes

$$
d s^{2}=\frac{2 C}{R_{0}}\left(-d t^{2}+\frac{1}{C} d \theta^{2}\right) .
$$

Since $\theta$ has the period $2 \pi$, the topology of the space with the metric (32) can be $S_{1}$. Therefore the topology of the space in the original BTZ model is the torus of $S_{1} \times S_{1}$. Note that Eq.(31) tells there is one to two correspondence between $\theta$ and $r$.

The functions $R$ and $S$ in (25) correspond to the eigenfunction with the eigenvalue $A(\overline{26})$ of the operator

$$
\Delta \equiv C \cosh ^{2}(r \sqrt{C}) \partial_{+} \partial_{-}
$$


which can be regarded as the Laplacian on two-dimensional Lorentzian hyperboloid, i.e., the Casimir operator of $S L(2, R)$. The operators of $S L(2, R)$ are given by

$$
L_{0}=\frac{1}{\sqrt{C}} \frac{\partial}{\partial t}, \quad L_{ \pm}=\frac{1}{\sqrt{C}} \mathrm{e}^{ \pm t \sqrt{C}}\left(\sinh (r \sqrt{C}) \frac{\partial}{\partial t} \pm \cosh (r \sqrt{C}) \frac{\partial}{\partial r}\right) .
$$

The operators $L_{0}$ and $L_{ \pm}$form the algebra $S L(2, R)$

$$
\left[L_{+}, L_{-}\right]=2 L_{0}, \quad\left[L_{0}, L_{ \pm}\right]= \pm L_{ \pm} .
$$

The eigenfunction of $\Delta$ in (25) is the sum of highest and lowest weight representation $\mathrm{e}^{ \pm t \alpha \sqrt{C}} \cosh ^{\alpha} r \sqrt{C}$. We now consider the following eigenfunction

$$
\begin{aligned}
S(t, r) & =\frac{Q}{4}\left(L_{+} \mathrm{e}^{t \alpha \sqrt{C}} \cosh ^{\alpha} r \sqrt{C}+L_{-} \mathrm{e}^{-t \alpha \sqrt{C}} \cosh ^{\alpha} r \sqrt{C}\right) \\
& =Q \sinh (t(\alpha+1) \sqrt{C}) \cosh ^{\alpha} r \sqrt{C} \sinh r \sqrt{C} .
\end{aligned}
$$

Since the evaporation of the black hole in [13] corresponds to the instable mode, we restrict $\alpha$ to be $0>\alpha>-1$. Then using the condition of horizon (27) we may estimate the asymptotic behavior of $S$

$$
\begin{aligned}
S(t, r(t)) & \stackrel{t \rightarrow 0}{\rightarrow} \pm(\alpha+1) C Q t^{2} \\
& \stackrel{t \rightarrow+\infty}{\rightarrow} \pm \frac{Q}{2^{\alpha+2}}\left(\frac{1-\alpha}{1+\alpha}\right)^{\frac{\alpha+1}{2}} \mathrm{e}^{t(\alpha+1)(\alpha+2) \sqrt{C}}
\end{aligned}
$$

Since the radius of the horizon is given by (29), the horizon corresponding to + sign in (37) grows up to infinity and that to - sign tends to zero when $t \rightarrow+\infty$ although the perturbation does not work when $S$ is large. The horizon of + sign corresponds to outer horizon and that of minus sign to inner horizon. Since there is one to two correspondence between the radial coordinates $r$ and $\theta$, there are two outer horizons and two inner ones. When we regard the model as 3D model, the handle with the topology of $S_{1} \times S_{1}$ is separated to two pieces when the two inner horizons tend to zero. Of course, this is qualitative discussion as perturbation should be small. Above result can be generalized if we use higher order eigenfunctions as perturbation

$$
S(t, r)=\frac{Q}{4}\left(\left(L_{+}\right)^{n} \mathrm{e}^{t \alpha \sqrt{C}} \cosh ^{\alpha} r \sqrt{C}+\left(L_{-}\right)^{n} \mathrm{e}^{-t \alpha \sqrt{C}} \cosh ^{\alpha} r \sqrt{C}\right) .
$$


Since the condition of the horizon (27) gives the $2 n$-th algebraic equation with respect to $\tanh r \sqrt{C}$, there can appear $2 n$ outer horizons and $2 n$ inner horizons and the spacetime could be disintegrated into $2 n$ pieces when the inner horizons tend to zero in accordance with proliferation proposal [13]. Note that horizon equation for the higher mode is $2 n$-th order polynomial with respect to $\tanh r \sqrt{C}$, which is the reason why it is expected that there can be $2 \times 2 n$ horizons. However, it is difficult to confirm that all solutions are real solutions.

6. In summary, we investigated quantum evolution of BTZ BH in large $N$ approximation. As we show the presence of instable modes in quantum spectrum manifests in anti-evaporation or possible topological proliferation. It is clear that in order to understand if above effects may really happen in early Universe one should select boundary conditions corresponding to BTZ BH formation and analyse above processes subject to such boundary conditions. Depending on the choice of boundary conditions they maybe compatible with anti-evaporation as it happens for Nariai anti-evaporating $\mathrm{BH}$ [1].

Our study is quite general and we may expect that similar results could be true for higher dimensional BHs described as product of BTZ sector and (anti)sphere. This is already confirmed [14] for 2D charged $\mathrm{BH}$ [15] (which is dual to 5D BH [16]) in the similar large $N$ approach used in the calculation of one-loop effective action for dilaton coupled scalars.

Acknoweledgments We thank R. Bousso for useful discussion.

\section{References}

[1] R. Bousso and S.W. Hawking, Phys.Rev. D56 (1997)7788.

[2] S. Nojiri and S.D. Odintsov, Mod.Phys.Lett. A12 (1997) 2083; Phys.Rev. D57 (1998) 2363.

[3] R. Bousso and S.W. Hawking, Phys.Rev. D57 (1998)2436.

[4] S. Nojiri and S.D. Odintsov, hep-th/9802160, hep-th/9804033. 
[5] M. Bañados, C. Teitelboim and J. Zanelli, Phys.Rev.Lett. 69 (1992) 1849.

[6] G.T. Horowitz and D.L. Welch, Phys.Rev.Lett. 71 (1993) 328; J.H. Horne and G.T. Horowitz, Nucl.Phys. B368 (1993) 444.

[7] A.A. Bytsenko, L. Vanzo ans S. Zerbini, Phys.Rev. D57 (1998) 4917; S. Nojiri, Int.J. of Mod.Phys. A12 (1997) 4309.

[8] S. Hyun, hep-th/9704005; K. Sfetsos and K. Skenderis, hepth/9711138.a

[9] G.T. Horowitz and A. Strominger, Phys.Rev.Lett. 77 (1996) 2368; G.T. Horowitz, D.A. Lowe and J.M. Maldacena, Phys.Rev.Lett. 77 (1996) 430.

[10] S. Carlip, Phys.Rev. D51 (1995) 632; D55 (1997) 878.

[11] J. Maldacena, hep-th/9711200; E. Witten, hep-th/9802116.a

[12] E. Elizalde, S. Naftulin and S.D. Odintsov, Phys.Rev. D49 (1994) 2852; T. Chiba and M. Siino, Mod.Phys.Lett. A12 (1997) 709; S. Ichinose, hepth/9707025; W. Kummer, H. Liebl and D.V. Vassilevich, Mod.Phys.Lett. A12 (1997) 2683; J.S. Dowker, hep-th/9802029.

[13] R. Bousso, hep-th/9805081.

[14] S. Nojiri and S.D. Odintsov, in preparation.

[15] M.D. McGuigan, C.R. Nappi and S.A. Yost, Nucl.Phys. B375 (1992) 421; G.W. Gibbons and M.J. Perry, Int.J.Mod.Phys. D1 (1992) 335.

[16] E. Teo, hep-th/9803064.

\section{Figure Captions}

Fig. $1 \frac{A}{C}$ (vertical line) versus $g \equiv 8 G N \mathrm{e}^{\phi_{0}}$ (horizontal line) when $\ln \mu^{2}=1$.

Fig.2a,b Two branches of $\frac{A}{C}$ (vertical line) versus $g \equiv 8 G N \mathrm{e}^{\phi_{0}}$ in $[0,1]$ and $\ln \mu^{2}$ in $[0,3]$. Fig.2a corresponds to the stable mode and $2 \mathrm{~b}$ to the instable one. 

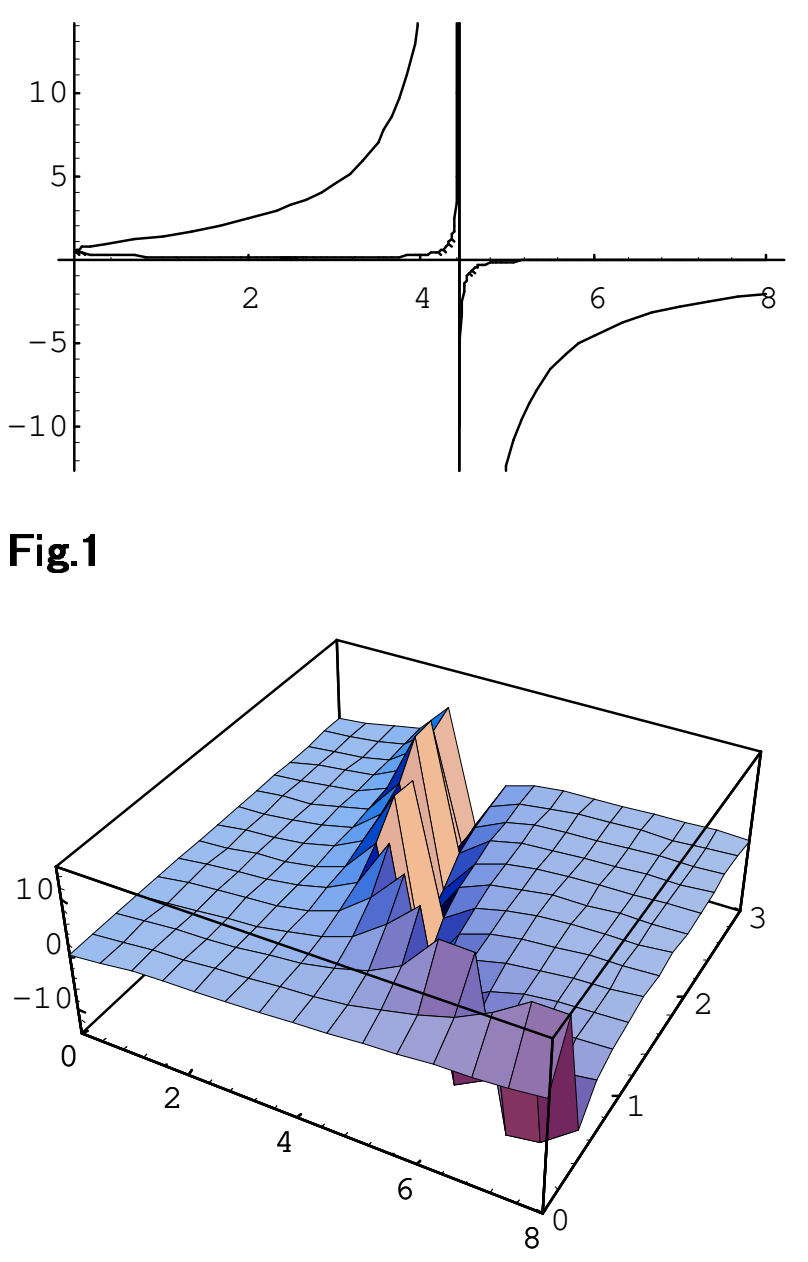

-

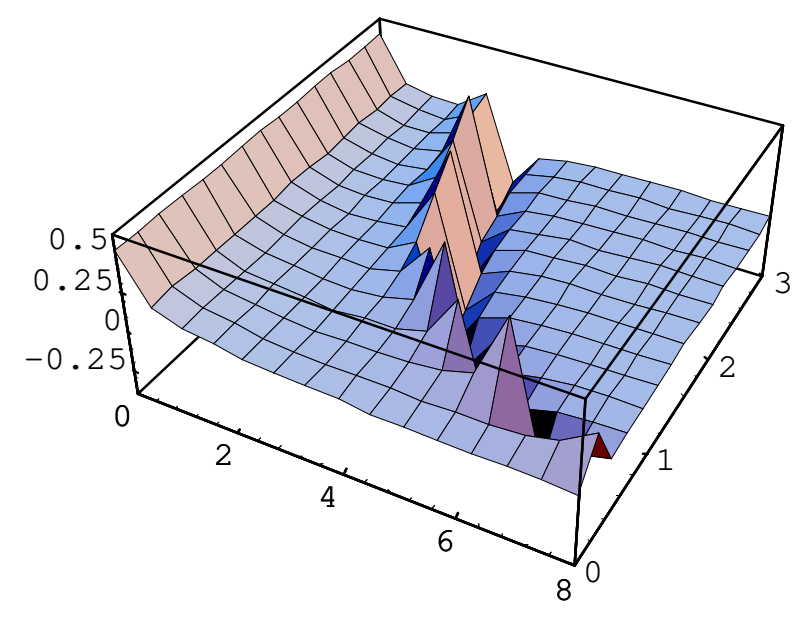

